\title{
ROJ
}

\section{The use of tissue fiducial markers in improving the accuracy of post-prostatectomy radiotherapy}

\author{
Michael Chao, FRANZCR ${ }^{1,2}$, Huong Ho, BSc, MSc${ }^{2}$, Daryl Lim Joon, FRANZCR', Yee Chan, FRACS', \\ Sandra Spencer, Dip App Sci Med Rad ${ }^{2}$, Michael Ng, FRANZCR², Jason Wasiak, PhD1, \\ Nathan Lawrentschuk, FRACS, PhD ${ }^{1}$, Kevin McMillan, FRACS ${ }^{3}$, Shomik Sengupta, FRACS, PhD ${ }^{3}$, \\ Alwin Tan, MBBS, FRACS ${ }^{4}$, George Koufogiannis, FRACS ${ }^{5}$, Margaret Cokelek, BSc ${ }^{2}$, \\ Farshad Foroudi, FRANZCR, Doc Med Sci ${ }^{1}$, Tristan-Scott Khong, BSc ${ }^{2}$, Damien Bolton, FRACS, PhD \\ ${ }^{1}$ The Austin Hospital, Heidelberg; ${ }^{2}$ Genesis Cancer Care Victoria, Melbourne; \\ ${ }^{3}$ Box Hill Hospital, Box Hill; ${ }^{4}$ The Bays Hospital, Mornington; ${ }^{5}$ The Ringwood Private Hospital, Ringwood East, Australia
}

Purpose: The aim of this retrospective study was to investigate the use of a radiopaque tissue fiducial marker (TFM) in the treatment of prostate cancer patients who undergo post-prostatectomy radiotherapy (PPRT). TFM safety, its role and benefit in quantifying the set-up uncertainties in patients undergoing PPRT image-guided radiotherapy were assessed.

Materials and Methods: A total of 45 consecutive PPRT patients underwent transperineal implantation of TFM at the level of vesicourethral anastomosis in the retrovesical tissue prior to intensity-modulated radiotherapy. Prostate bed motion was calculated by measuring the position of the TFM relative to the pelvic bony anatomy on daily cone-beam computed tomography. The stability and visibility of the TFM were assessed in the initial 10 patients.

Results: No postoperative complications were recorded. A total of 3,500 images were analysed. The calculated prostate bed motion for bony landmark matching relative to TFM were $2.25 \mathrm{~mm}$ in the left-right, $5.89 \mathrm{~mm}$ in the superior-inferior, and $6.59 \mathrm{~mm}$ in the anterior-posterior directions. A significant $36 \%$ reduction in the mean volume of rectum receiving $70 \mathrm{~Gy}\left(\mathrm{rV}_{70}\right)$ was achieved for a uniform planning target volume (PTV) margin of $7 \mathrm{~mm}$ compared with the Australian and New Zealand Faculty of Radiation Oncology Genito-Urinary Group recommended PTV margin of $10 \mathrm{~mm}$.

Conclusion: The use of TFM was safe and can potentially eliminate set-up errors associated with bony landmark matching, thereby allowing for tighter PTV margins and a consequent favourable reduction in dose delivered to the bladder and rectum, with potential improvements in toxicities.

Keywords: Fiducial marker, Image-guided radiotherapy, Intensity-modulated radiotherapy, Prostatectomy, Prostate cancer

\section{Introduction}

Although consensus guidelines are available to help define the clinical target volume (CTV), one of the technical challenges that remain is defining the optimal planning target volume (PTV) expansion. The current post-prostatectomy consensus

Received 19 December 2018, Revised 10 January 2019, Accepted 12 February 2019.

Correspondence: Michael Chao, Department of Radiation Oncology, The Austin Hospital, 145, Studley Rd. Heidelberg, Victoria 3084, Australia. Tel: +61-03-94962800, Fax: +61-03-88703308, E-mail: Michael.chao@genesiscare.com (http://orcid. org/0000-0002-3497-3746)

(c) This is an Open Access article distributed under the terms of the Creative Commons Attribution Non-Commercial License (http://creativecommons.org/ licenses/by-nc/4.0/) which permits unrestricted non-commercial use, distribution, and reproduction in any medium, provided the original work is properly cited.

www.e-roj.org 
guidelines of the Australian and New Zealand Faculty of Radiation Oncology Genito-Urinary Group (FROGG) recommend a uniform 10-mm margin in all directions to account for daily treatment set uncertainties [1]. As rectal dose constraints can be difficult to achieve, a caveat of a $5-\mathrm{mm}$ posterior expansion is deemed acceptable in these situations. However, this has the potential of increasing the risk of geographic misses.

The use of fiducial markers such as gold seeds and surgical clips placed in the prostate bed has been reported in the literature [2-7]. Although surgical clips have the advantage of being non-invasive, some studies have found them difficult to match to because of the varying number and asymmetric shape $[5,7]$. The use of gold seeds has been found to be reliable as they are easily identifiable, stable and representative of the prostate bed [5-7]. Over the past few years, a radiopaque hydrogel tissue fiducial marker (TFM) called TracelT (Augmenix Inc., Waltham, MA, USA), has gained considerable interest as a soft tissue marker in assisting with target delineation and treatment verification $[8,9]$. TracelT is a particulated injection that is visible on magnetic resonance imaging (MRI), computer tomography (CT), and cone beam computer tomography (CBCT). In addition, it has no CT artefact and creates no dose perturbation.

In this study, the primary endpoint was to investigate the safety, visibility and stability of TFM in its role as a fiducial marker. The secondary endpoints were to determine the required PTV margin expansion using conventional bony landmark versus TFM image-guided radiotherapy (IGRT) and to determine the dosimetric impact of a tighter PTV expansion on rectum and bladder organs at risk (OAR) when TFM IGRT is used.

\section{Materials and Methods}

\section{Study design}

A retrospective review of 45 patients who underwent TFM implantation prior to a course of salvage post-prostatectomy radiation therapy (PPRT) was performed. Institutional Review Board approval was obtained prior to commencement of this study. All participants provided written medical informed consent prior to undergoing any therapeutic procedure.

\section{Participants}

Forty-five consecutive patients referred to one radiation oncologist who commenced PPRT at Genesis Cancer Care Victoria, Melbourne, Australia between January 2016 and
November 2017 were included in this study. Eligible patients were consecutive men $>18$ years of age with histologically confirmed prostate cancer post radical prostatectomy (RP) requiring salvage PPRT. These patients had either a persistent or rising prostate specific antigen (PSA) post RP with or without positive surgical margins, extraprostatic extension or seminal vesicle invasion. The exclusion criteria included known allergy to iodine or contrast, known metabolic disorder, distant metastatic disease, unilateral or bilateral total hip replacement and previous pelvic RT.

\section{TFM (TracelT) injection}

Implantation of TFM was performed by one radiation oncologist specialised in prostate brachytherapy. Once the patient was anaesthetised, intravenous prophylactic antibiotic was given. The patient was then set up in the dorsal lithotomy position. Once the patient's perineum, supra-pubic and lower anterior abdomen area were prepped with betadine, a 16F indwelling catheter (IDC) was inserted into the bladder. The IDC balloon was filled with $10 \mathrm{~mL}$ of normal saline. Utilising the same brachytherapy principle and equipment, a transrectal ultrasound (TRUS) probe was inserted into the rectum to visualise the bladder, urethra and prostate bed. Gentle traction on the IDC balloon seated at the bladder neck would echographically define the anatomy of the vesicourethral anastomosis (VUA). An 18-gauge disposable brachytherapy grid was attached onto the top of the brachytherapy stepper to help guide the injection of TFM. Using a 18-gauge spinal needle, an average of $0.5 \mathrm{~mL}$ of TFM was then injected transperineally on either side of the VUA into the retrovesical tissue (Fig. 1A, 1B). The time taken to perform this procedure was on average 15 minutes. A total of two TFM blebs were injected into each patient. Patients were assessed immediately post-operatively and approximately $5-7$ days later to determine the incidence and nature of adverse effects related to the TFM implant.

\section{Treatment planning procedure}

A pelvic planning CT scan for intensity-modulated radiotherapy or volumetric modulated arc therapy was performed approximately 5-7 days post-TFM implantation. All patients were scanned in the supine position with strict instructions for both bladder and bowel preparation to follow prior to CT simulation and daily treatments. The patients were instructed to empty their bladder and then drink $500 \mathrm{~mL}$ of water 1 hour prior to CT simulation and subsequent treatments. An enema was also used prior to CT simulation and for the first 

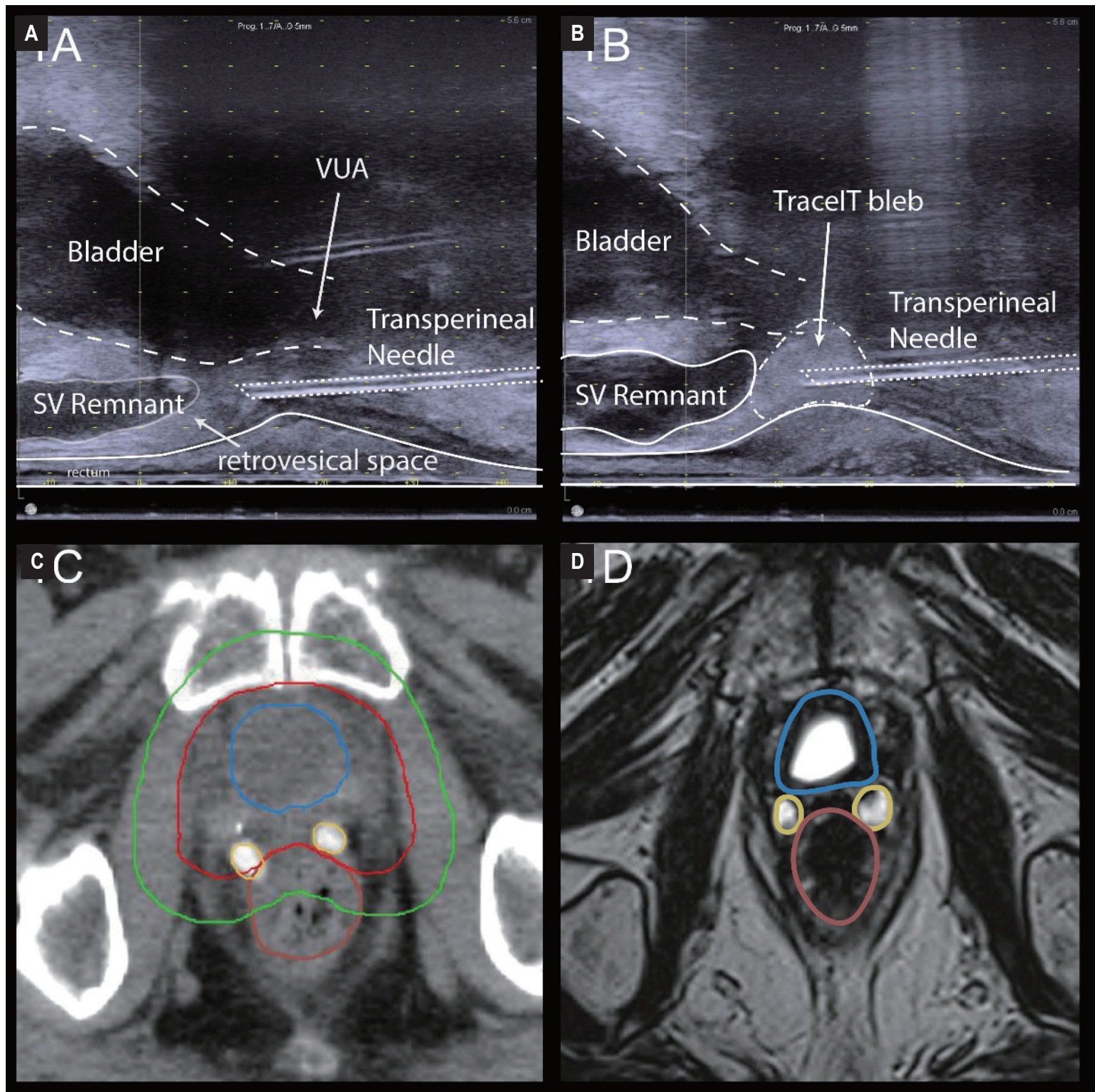

Fig. 1. (A) A transrectal ultrasound sagittal image of the prostate bed angled at approximately $3^{\circ}-5^{\circ}$ off centre. The bladder, remnant seminal vesicle (SV), rectum, position of vesicourethral anastomosis (VUA) and retrovesical space are outlined. The transperineal needle is advanced into the retrovesical space superior to the VUA. (B) The outlined TracelT bleb injected into the retrovesical tissue. (C) A transverse planning computed tomography slice at the level of the TracelT blebs. The bladder is outlined in blue, rectum in brown, TracelT bleb in yellow, the clinical target volume in red, and the planning target volume in green. (D) A transverse magnetic resonance imaging slice at the level of the TracelT blebs. The bladder is outlined in blue, rectum in brown and the TracelT blebs in yellow. The TracelT blebs are positioned in the retrovesical tissues.

10 fractions of PPRT. This may be continued if the patient failed to maintain an empty rectum during the course of PPRT. CT simulation was performed using a Philips Brilliance Big
Bore CT scanner (Philips Medical Systems, Fitchburg, WI, USA) with 3-mm slice thickness. The data sets were electronically transferred to the MIMS v6.7.5 (MIM Software Inc. Cleveland, 


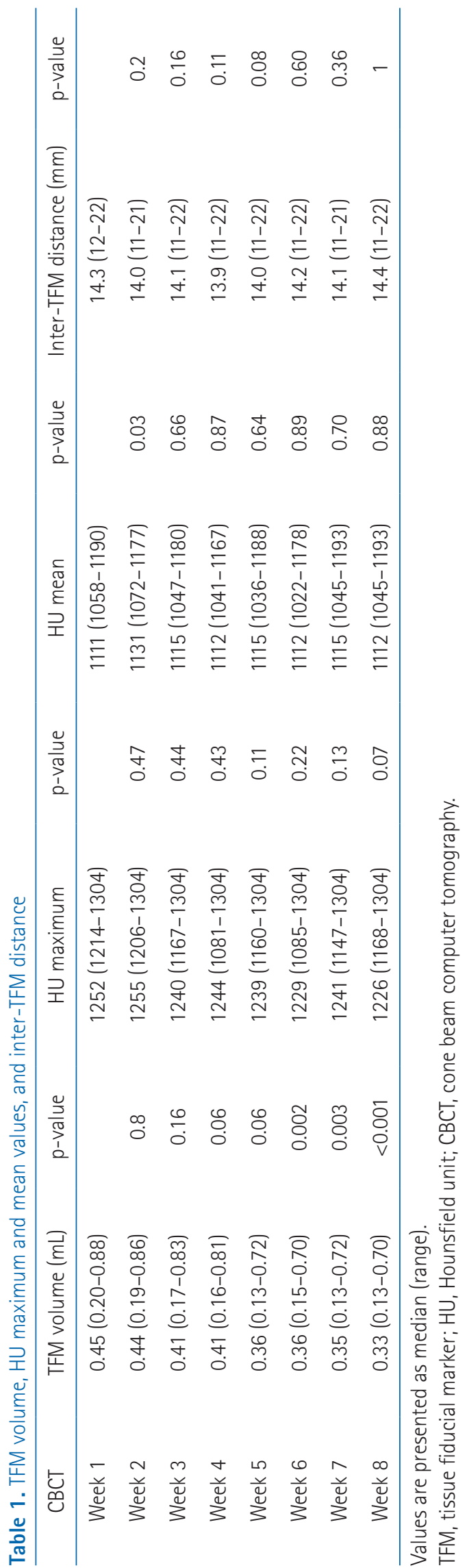

$\mathrm{OH}$, USA) for contouring prior to transferring to Pinnacle v9.8 (Philips Medical Systems) treatment planning system for dosimetry.

The prostate bed or CTV was contoured according to the FROGG guidelines [1]. The normal tissues contoured included the bladder, rectum, VUA, both femoral heads and penile bulb. The implanted TFM were contoured separately and labelled as 'TFM 1' and 'TFM 2' (Fig. 1C, 1D). A 7-mm uniform expansion was applied to the CTV to create the PTV according to our departmental protocol. All patients were treated daily, 5 days per week on a Varian True Beam linear accelerator equipped with kilovoltage $(\mathrm{kV}) \mathrm{CBCT}$ capabilities. A prescription dose of $70.2 \mathrm{~Gy}$ in 39 fractions was delivered to the PTV, covered by at least $95 \%$ of the prescription dose. Rectal dose constraint objectives for the volume of rectum receiving $70 \mathrm{~Gy}\left(\mathrm{rV}_{70}\right), 60$ Gy $\left(\mathrm{rV}_{60}\right), 50 \mathrm{~Gy}\left(\mathrm{rV}_{50}\right), 40 \mathrm{~Gy}\left(\mathrm{rV}_{40}\right)$, and $30 \mathrm{~Gy}\left(\mathrm{rV}_{30}\right)$ were $20 \%$, $35 \%, 50 \%, 60 \%$, and $70 \%$ of the rectal volume, respectively. Bladder dose constraints objectives for the volume of bladder receiving $65 \mathrm{~Gy}_{(}\left(\mathrm{bV}_{65}\right)$ and $70 \mathrm{~Gy}\left(\mathrm{bV}_{70}\right)$ were $50 \%$ and $35 \%$. The mean penile bulb dose was kept below $25 \mathrm{~Gy}$ if achievable.

\section{Matching guidelines}

Our department imaging protocol for PPRT patients was daily on-line CBCT matched to the TFM using the soft tissue alignment algorithm. The patient was initially set up to skin tattoos, followed by daily on-line CBCT match to the TFM and off-line matching to bony landmark anatomy. Off-sets relative to skin tattoos were recorded in three directions: $L R=$ left $(+)$ / right (-), $\mathrm{SI}=$ superior $(+) /$ inferior $(-)$, and $\mathrm{AP}=$ anterior $(+) /$ posterior (-). All shifts were recorded in $\mathrm{mm}$. No couch rotation shifts were performed. Mean and standard deviation (SD) of the shift differences were calculated for all 45 patients over all measured fractions for both bony landmark and TFM IGRT. The bony landmark IGRT represented the set-up error and was calculated from the shift of skin tattoos to bony anatomy localisation. The TFM IGRT represented the total position error and was calculated from the shift of skin tattoos to the TFM. The difference in shifts recorded between bony landmark and TFM IGRT represented the improvement in treatment accuracy or inter-fractional prostate bed motion (PBM) when TFM IGRT was compared to bony landmark IGRT. This was used to derive the overall mean systematic error (OM), SD of systematic error $(\Sigma)$, and SD of random error $(\sigma)$, as previously described by van Herk [10]. The PTV margins were defined in three separate directions ( $L R, S I$, and AP axes) from the entire patient data using the equation formula of $2.5 \Sigma+0.7 \sigma$, which is designed to ensure that $90 \%$ of patients in the population 
Table 2. Magnitude of shifts for TFM and bony landmark IGRT

\begin{tabular}{|c|c|c|c|c|c|c|}
\hline & \multicolumn{2}{|c|}{$\mathrm{LR}$} & \multicolumn{2}{|c|}{$\mathrm{SI}$} & \multicolumn{2}{|c|}{$A P$} \\
\hline & TFM & Bony & TFM & Bony & TFM & Bony \\
\hline Mean (mm) & 0.25 & 0.39 & -0.45 & -0.51 & 1.92 & 1.10 \\
\hline Systematic error (mm) & 1.96 & 1.82 & 1.97 & 1.83 & 2.96 & 3.10 \\
\hline Random error (mm) & 2.89 & 2.79 & 3.05 & 2.95 & 3.41 & 3.38 \\
\hline
\end{tabular}

TFM, tissue fiducial marker; IGRT, image-guided radiotherapy; LR, left-right; SI, superior-inferior; AP, anterior-posterior.

Table 3. Inter-fractional prostate bed motion: random and systematic errors

\begin{tabular}{lccc}
\hline & LR & SI & AP \\
\hline Overall mean $(\mathrm{mm})$ & -0.14 & 0.06 & 0.82 \\
SD mean $\Sigma(\mathrm{mm})$ & 0.53 & 1.72 & 1.81 \\
RMS SD $\sigma(\mathrm{mm})$ & 1.33 & 2.26 & 2.94 \\
Margin $=2.5 \Sigma+0.7 \sigma(\mathrm{mm})$ & 2.25 & 5.89 & 6.59 \\
\hline
\end{tabular}

Overall mean, group systematic errors; SD mean, standard deviation of systematic error; RMS SD, standard deviation of random error; $L R$, left-right; SI, superior-inferior; $A P$, anterior-posterior.

receive a minimum cumulative CTV dose of at least $95 \%$ of the prescribed dose.

\section{TFM stability, visibility assessment}

The visibility and volume stability of the TFMs were assessed in the first 10 patients. The TFM volume in $\mathrm{mL}$, its Hounsfield (HU) maximum and mean values, as well as the inter-fraction distance between the two TFMs placed in each patient were measured. Weekly CBCT images from week 1 to week 8 were imported back to the Pinnacle v.9.8 treatment planning system (TPS) and each bleb were contoured and measured. The change in TFM volume, its HU maximum and mean values and interfraction TFM distance from week 1 to week 8 CBCT were assessed for significance using the paired t-test.

\section{Planning margins assessment}

To further assess the benefits of TFM and its impact on rectal, bladder and penile bulb dosimetry, the first ten consecutive PPRT patients also had a second treatment plan generated with the FROGG recommended consensus PTV expansion of 10 $\mathrm{mm}$ around the CTV.

\section{Results}

IThe median age at commencement of PPRT was 68 years (range, 48 to 76 years). The majority of patients (31) had Gleason 7 adenocarcinoma (69\%), 1 had Gleason 8 adenocarcinoma, and 13 others had Gleason 9 adenocarcinoma
(29\%). The primary T staging were pT2 (24\%) and pT3 (76\%). All patients had detectable PSA levels prior to PPRT, with a level of $<0.2$ for $62 \%$ and $>0.2$ for $38 \%$. No patients developed bleeding, infection, allergic reactions, urinary retention, rectal perforation or systemic embolization following TFM implantation.

\section{TFM stability, visibility and migration}

A total of $80 \mathrm{CT}$ and CBCT images were reviewed for the 10 patients assessed. The TFM volume in $\mathrm{mL}$, its $\mathrm{HU}$ maximum and mean values as well as interfraction TFM distance is found in Table 1. There was no statistically significant difference found except for change in TFM volume in $\mathrm{mL}$ from week 6 to week 8. The mean TFM volume was $0.45 \mathrm{~mL}$ at week 1 of PPRT, decreasing to $0.33 \mathrm{~mL}$ by week 8 of PPRT. This represented a volume loss of $27 \%$ over the 3 -month period from TFM injection to completion of PPRT. Although there was a significant reduction from week 6 onwards, the TFM remained highly discernible on soft tissue windows as demonstrated by its HU maximum and mean values. The HU maximum and mean values remained stable.

To assess migration of the TFM implanted into the prostate bed, the variations in the TFM distance between fractions and over the course of the treatment were measured. The interfraction variation in TFM distance was very small and ranged between 0 to $0.4 \mathrm{~mm}$ (mean). As such the migration of the TFM over the course of PPRT was minimal and not significant. 
Table 4. Different PTV margins and impact on OARs

\begin{tabular}{|c|c|c|c|c|}
\hline OAR & 10-mm PTV margin (\%) & 7-mm PTV margin (\%) & \%Reduction & $p$-value \\
\hline$r V_{30}<70 \%$ & $77(51-94)$ & $59(42-79)$ & 23 & $<0.001$ \\
\hline $\mathrm{rV}_{40}<60 \%$ & $68(48-88)$ & $48(37-68)$ & 29 & $<0.001$ \\
\hline $\mathrm{rV}_{50}<50 \%$ & $60(43-81)$ & $40(31-58)$ & 34 & $<0.001$ \\
\hline$r V_{60}<35 \%$ & $51(37-71)$ & $31(23-48)$ & 39 & $<0.001$ \\
\hline$r V_{70}<20 \%$ & $22(11-43)$ & $13(5-24)$ & 36 & $<0.001$ \\
\hline $\mathrm{bV}_{65}<50 \%$ & $40(12-60)$ & $32(9-49)$ & 19.5 & $<0.001$ \\
\hline $\mathrm{bV}_{70}<35 \%$ & $29(9-47)$ & $22(5-33)$ & 23 & $<0.001$ \\
\hline Penile bulb (Gy) & $30(14-46)$ & $22.5(9-37)$ & 25 & $<0.001$ \\
\hline
\end{tabular}

Values are presented as mean (range).

PTV, planning target volume; OAR, organ at risk; $r V n$, volume of rectum receiving dose $n$; $b V n$, volume of bladder receiving dose $n$.

2. Inter-fractional prostate bed motion and set up errors A total of 3,500 images were assessed. Although the magnitude of shifts was similar for both bony and TFM in both the $L R$ and SI directions, the largest magnitude of error was recorded in the AP direction (Table 2).

Inter-fraction PBM which represents the difference between bony landmark IGRT and TFM IGRT in terms of OM, $\Sigma$, and $\sigma$ of $\mathrm{PBM}$ in $\mathrm{AP}, \mathrm{SI}$, and $\mathrm{LR}$ directions is presented in Table 3. Based on the results above, if the patients were matched according to bony anatomy, margins of $2.25 \mathrm{~mm}$ along the $L R$ axis, 5.89 $\mathrm{mm}$ along the $\mathrm{SI}$ axis, and $6.59 \mathrm{~mm}$ along the AP axis would be required to ensure the minimum dose to the CTV is at least $95 \%$ of the prescription dose in $90 \%$ of patients.

\section{Impact of $10 \mathrm{~mm}$ vs. $7 \mathrm{~mm}$ PTV on organ at risk}

Table 4 clearly demonstrates a statistically significant improvement in all rectal, bladder and penile bulb dose constraints when a 7-mm PTV margin is compared with a 10$\mathrm{mm}$ PTV margin. A significant 36\% reduction in mean $r V_{70}$ was achieved. In the 10 patients analysed with a 10-mm PTV margin, rectal dose constraints were not met in 6 patients for $r V_{70}$, in all 10 patients for $r V_{60}$, in 8 patients for $r V_{50}$, in 7 patients for $r V_{40}$, and in 5 patients for $r V_{30}$. All rectal dose constraints were met in 9 patients with a 7-mm PTV margin. The bladder dose constraints for $b_{65}$ and $b V_{70}$ were not met in 4 patients with a 10-mm PTV margin but achieved in all 10 patients with a 7-mm PTV margin. In addition, an average of $33 \%$ reduction was achieved for the mean penile bulb dose for $7 \mathrm{~mm}$ margin instead of $10 \mathrm{~mm}$ margin.

\section{Discussion and Conclusion}

The use of TracelT as a TFM in our study was found to be safe and efficacious. No significant adverse events were reported.

Prior studies have investigated the role of fiducial markers in PPRT used gold seeds or surgical clips as surrogate fiducial markers. Both have been found to be reliable markers, being easily identifiable, stable and representative of the target volume. Although surgical clips have the advantage of being non-invasive, some studies have found them difficult to match to $[5,7]$. In our study, we used a radiopaque hydrogel TFM called TracelT. The TFM was implanted into the prostate bed and remained fixed within the postoperative tissues, serving reliably as fiducials to target the CTV over the course of PPRT. The TFM implanted in our study were found to be stable with negligible migration reported over the course of PPRT. This compared favourably to the use of gold seeds [5-7]. Although we detected a $27 \%$ loss of TFM volume during the course of their treatment, the visibility of the TFM remained unaffected with persistently high mean and maximum HU. The TFM was clearly visible on CBCT with no issues in its identification and daily matching on CBCT.

Variability was seen between bony anatomy and TFM matching in all axes, with the largest magnitude of shift in the AP plane and the least in the LR plane. We recorded an overall means (SD) of -0.14 (0.53) mm, 0.06 (1.72) mm, and 0.82 (1.81) $\mathrm{mm}$ in the $L R, S I$, and AP planes, respectively. The SD of the overall means in the SI and AP axis is also larger, meaning greater interfraction variation in these planes compared to the LR plane. These daily variations are secondary to the impact of changes in daily bladder and rectal filling leading to the differences in the position of the prostate bed and hence CTV. Nevertheless, we recorded a smaller magnitude of error compared to previously published studies $[2,4,7]$, no doubt attributed to our strict departmental CBCT imaging protocol. Our patients were required to comply with a treatment $\mathrm{CBCT}$ 
bladder volume greater than 50\% of planning CT volume, and a treatment CBCT rectal size that was within $\pm 2 \mathrm{~cm}$ of the planning rectal CT size. Feedback was given daily to our patients and if they failed to meet our image matching protocol, they were duly removed from the treatment couch to allow for correction of bladder filling and rectal emptying before a later attempt at treatment.

The minimum PTV margin required for PBM in our study was $2.25 \mathrm{~mm}$ along the $L R, 5.89 \mathrm{~mm}$ along the SI, and $6.59 \mathrm{~mm}$ along the AP axes. The FROGG recommended PTV expansion of $10 \mathrm{~mm}$ was adequate to minimise any potential geographic misses with a minimal IGRT standard of bony landmark matching only. Our calculated PBM with the use of TFM and CBCT was similar to the results from Alander et al. [5] who used a combination of gold seed fiducials and CBCT $(1.4 \mathrm{~mm}$ $L R, 5.9 \mathrm{~mm} \mathrm{SI}$, and $5.9 \mathrm{~mm} \mathrm{AP)}$ and Huang et al. [3] who used a combination of clips and CBCT (3.24 mm LR, $5.49 \mathrm{~mm} \mathrm{SI}$, and $8.36 \mathrm{~mm} \mathrm{AP)}$. This was in contrast with the use of gold seed fiducials or surgical clips with orthogonal $\mathrm{kV}$ images instead of CBCT where the PTV margins were slightly larger $[4,7]$.

The use of image guidance with fiducial markers and CBCT in PPRT can support the adoption of tighter PTV margins as the risk of geographic misses is minimised. Compared to a $10-\mathrm{mm}$ uniform PTV margin, we observed significant radiation dose reduction in both bladder and rectal volumes as well as a 33\% radiation dose reduction in penile bulb dose for our 7-mm PTV margin plan. This is particularly important for our rectal OAR, as it significantly reduced rectal irradiation in the high dose region from $r V_{50}$ to $r V_{70}$. This is critical because rectal toxicity is correlated with the volume of rectum receiving a particular threshold dose of radiation, particularly $r V_{70}[11,12]$. Therefore, the use of tighter PTV margins can further reduce rectal radiation doses, thus potentially reducing late gastrointestinal toxicity. In addition, smaller PTV margins may allow for a safer means of dose escalation, which can lead to better biochemical control [13-15].

However, our study does have several imitations. Our TFM were implanted at the level of the VUA in the lower prostate bed. The upper prostate bed can move independently of the lower prostate bed due to the close proximity of the bladder and rectum [2]. A pragmatic decision was made to implant the TFM at the level of the VUA in the lower prostate bed as this is the area at highest risk of recurrence post prostatectomy [1621]. As such this area needs to be targeted as accurately as possible. The upper prostate bed which can tilt in the AP plane is very difficult to correct for, as most treatment couches do not have the ability to tilt or only possess a limited range to do so. Instead we relied on a strict departmental CBCT protocol to reduce the daily variation in bladder and rectal filling to minimise the tilt. In addition, we had a tight imaging tolerance threshold to take the patient off the treatment couch to make corrections to bladder and rectal filling if the parameters were not met. Secondly, we only implanted two fiducial markers into the prostate bed. However, the use of two fiducial markers is not unique and has been found to be effective [6]. It will not allow us to capture potential rotational errors, however a previous study has shown rotational shifts to be very small, and therefore would not be expected to contribute significantly to target motion [22]. Finally, we did not measure intrafractional PBM. Huang et al. [3] who calculated intrafractional PBM in his study showed potential shifts of 2.8 $\mathrm{mm}$ along the LR, $3.9 \mathrm{~mm}$ along the $\mathrm{SI}$, and $4.3 \mathrm{~mm}$ along the AP axes, respectively. However, our departmental PTV margin of $7 \mathrm{~mm}$ despite the use of image guidance with TFM and CBCT is sufficient to deal with any intrafractional PBM. As such, we would hesitate against reducing the PTV margin any further.

In conclusion, to our knowledge, this is the first and largest series assessing and supporting the use of a novel TFM with CBCT image guidance in PPRT. The use of TracelT as a TFM was found to be safe and effective. It had no CT artefact, was stable and highly visible on CT and CBCT. PBM is independent of pelvic bone anatomy. With a PTV margin of at least $6.59 \mathrm{~mm}$ required in the AP direction with bony landmark matching, the use of TFM can improve the set-up accuracy, ensuring CTV coverage and reducing bladder and rectal dose despite tighter PTV margins.

\section{Conflict of Interest}

No potential conflict of interest relevant to this article was reported.

\section{References}

1. Sidhom MA, Kneebone $A B$, Lehman $M$, et al. Postprostatectomy radiation therapy: consensus guidelines of the Australian and New Zealand Radiation Oncology GenitoUrinary Group. Radiother Oncol 2008;88:10-9.

2. Bell LJ, Cox J, Eade T, Rinks M, Kneebone A. Prostate bed motion may cause geographic miss in post-prostatectomy image-guided intensity-modulated radiotherapy. J Med Imaging Radiat Oncol 2013;57:725-32.

3. Huang K, Palma DA, Scott D, et al. Inter- and intrafraction 
uncertainty in prostate bed image-guided radiotherapy. Int J Radiat Oncol Biol Phys 2012;84:402-7.

4. Song S, Yenice KM, Kopec M, Liauw SL. Image-guided radiotherapy using surgical clips as fiducial markers after prostatectomy: a report of total setup error, required PTV expansion, and dosimetric implications. Radiother Oncol 2012;103:270-4.

5. Alander E, Visapaa H, Kouri M, Keyrilainen J, Saarilahti K, Tenhunen M. Gold seed fiducials in analysis of linear and rotational displacement of the prostate bed. Radiother Oncol 2014;110:256-60.

6. Schiffner DC, Gottschalk AR, Lometti M, et al. Daily electronic portal imaging of implanted gold seed fiducials in patients undergoing radiotherapy after radical prostatectomy. Int J Radiat Oncol Biol Phys 2007;67:610-9.

7. Fortin I, Carrier JF, Beauchemin MC, Beliveau-Nadeau D, Delouya G, Taussky D. Using fiducial markers in the prostate bed in postprostatectomy external beam radiation therapy improves accuracy over surgical clips. Strahlenther Onkol 2014;190:467-71.

8. Bair RJ, Bair E, Viswanathan AN. A radiopaque polymer hydrogel used as afiducial marker in gynecologic-cancer patients receiving brachytherapy. Brachytherapy 2015;14:87680.

9. Chao M, Bolton D, Subramanian B, et al. The use of injectable TracelT tissue marker to delineate bladder tumor volume for targeted partial bladder IMRT. Int J Urol 2016;23(Suppl 1):47.

10. van Herk M. Errors and margins in radiotherapy. Semin Radiat Oncol 2004;14:52-64.

11. Huang EH, Pollack $A$, Levy $L$, et al. Late rectal toxicity: dosevolume effects of conformal radiotherapy for prostate cancer. Int J Radiat Oncol Biol Phys 2002;54:1314-21.

12. Michalski JM, Yan $Y$, Watkins-Bruner $D$, et al. Preliminary toxicity analysis of 3-dimensional conformal radiation therapy versus intensity modulated radiation therapy on the high-dose arm of the Radiation Therapy Oncology Group 0126 prostate cancer trial. Int J Radiat Oncol Biol Phys 2013;87:932-8.

13. Anscher MS, Clough R, Dodge R. Radiotherapy for a rising prostate-specific antigen after radical prostatectomy: the first
10 years. Int J Radiat Oncol Biol Phys 2000;48:369-75.

14. Cozzarini C, Montorsi F, Fiorino C, et al. Need for high radiation dose (>or $=70 \mathrm{gy}$ ) in early postoperative irradiation after radical prostatectomy: a single-institution analysis of 334 high-risk, node-negative patients. Int J Radiat Oncol Biol Phys 2009;75:966-74.

15. Valicenti RK, Gomella LG, Ismail M, Mulholland SG, Petersen $\mathrm{RO}$, Corn BW. Effect of higher radiation dose on biochemical control after radical prostatectomy for PT3NO prostate cancer. Int J Radiat Oncol Biol Phys 1998;42:501-6.

16. Connolly JA, Shinohara K, Presti JC Jr, Carroll PR. Local recurrence after radical prostatectomy: characteristics in size, location, and relationship to prostate-specific antigen and surgical margins. Urology 1996;47:225-31.

17. Leventis AK, Shariat SF, Slawin KM. Local recurrence after radical prostatectomy: correlation of US features with prostatic fossa biopsy findings. Radiology 2001;219:432-9.

18. Scattoni $V$, Roscigno $M$, Raber $M$, et al. Multiple vesicourethral biopsies following radical prostatectomy: the predictive roles of TRUS, DRE, PSA and the pathological stage. Eur Urol 2003:44:407-14.

19. Liauw SL, Pitroda SP, Eggener SE, et al. Evaluation of the prostate bed for local recurrence after radical prostatectomy using endorectal magnetic resonance imaging. Int J Radiat Oncol Biol Phys 2013;85:378-84.

20. Miralbell R, Vees $H_{1}$ Lozano J, et al. Endorectal MRI assessment of local relapse after surgery for prostate cancer: a model to define treatment field guidelines for adjuvant radiotherapy in patients at high risk for local failure. Int J Radiat Oncol Biol Phys 2007;67:356-61.

21. Kitajima K, Murphy RC, Nathan MA, et al. Detection of recurrent prostate cancer after radical prostatectomy: comparison of $11 \mathrm{C}$-choline PET/CT with pelvic multiparametric MR imaging with endorectal coil. J Nucl Med 2014;55:223-32.

22. Broggi $S$, Cozzarini $C$, Fiorino $C_{\text {, }}$ et al. Modeling set-up error by daily MVCT for prostate adjuvant treatment delivered in 20 fractions: Implications for the assessment of the optimal correction strategies. Radiother Oncol 2009;93:246-52. 